\title{
Adrenal Insufficiency In Critically Ill Patients
}

\author{
S M Ashrafuzzaman
}

\begin{abstract}
Adrenal insufficiency means hypo function of the adrenal cortex, usually resulting in low glucocorticoid level and it may be associated with low mineralocorticoid, rarely low adrenal androgen level. It can be categorized into two types: primary and secondary. Primary adrenal insufficiency or Addison's disease is associated with low cortisol and high ACTH level. Secondary/tertiary adrenal insufficiency is due to pituitary or hypothalamic disorders and is associated with both low cortisol and ACTH level. Among critically ill patients (CIP) adrenal insufficiency is not uncommon. The reported incidence of adrenal insufficiency varies greatly depending on the population of critically ill patients studied, the type of test, cut off levels used, and the severity of illness. Several studies have shown increased mortality in patients with very low or very high baseline cortisol levels. Manifestations of adrenal insufficiency in the critically ill patient are numerous and nonspecific, so clinicians are urged to have a high index of suspicion while taking history and doing physical examination and be alert to important diagnostic clues, such as hyponatremia, hyperkalemia, and hypotension, that are refractory to fluids and vasopressor without any clear causation.

In current literature there is no consensus level of cortisol (basal/random/stimulated) in critically ill subjects. But it is shown that both high and low cortisol level is associated with increased mortality. In one study Basal Serum Cortisol $<414 \mathrm{nmol} / \mathrm{L}$ and $>696 \mathrm{nmol} / \mathrm{L}$ is shown as indicative of higher risk among critically ill patients. Even with Septicemia or ARDS all subjects don't suffer from adrenal insufficiency often termed relative adrenal insufficiency or critical illness associated adrenal insufficiency. It has been observed that short term low dose IV hydrocortisone may be beneficial in selective group of patients in intensive care unit (ICU) with critical illness.

Diagnosis of adrenal insufficiency associated with critical illness is still challenging for physicians working in ICU. Treatment should be started without delay in emergency situation with Injection Hydrocortisone intravenously or even intramuscularly. Since the condition appears to be common in patients with septic shock, clinicians should have a high index of suspicion for its occurrence in critically ill patients with persistent hypotension despite adequate fluid resuscitation and/or poor hemodynamic response to vasopressor. Adrenal insufficiency associated with other illnesses in ICU are attributed to previous primary or secondary adrenal insufficiency. Treatment with physiologic doses of corticosteroids should be started as soon as possible since short-term treatment carries very few risks and has been shown to decrease both morbidity and mortality. Only suspected cases should be evaluated and could be treated with 100-200 mg Hydrocortisone in divided doses for 5-7 days. Glucocorticoid cannot be recommended as a routine adjuvant therapy in all cases of septic shock or ARDS. But glucocorticoid earned its position among other rescue strategies in subgroups of ICU patients with the highest mortality risk. Steroid use is an art and needs to be used by experienced physician. Otherwise it may do more harm than benefit.
\end{abstract}

\section{Introduction}

Primary adrenal insufficiency was first recognized by Addison in 1844 and described in 1855 in one of the classic articles in medicine. ${ }^{1}$ Subnormal cortisol production is the hallmark of this condition. This condition is also observed among critically ill patients specially with sepsis or septic shock and it is different from primary or secondary adrenal insufficiency (AI). Any stress, trauma or even infection usually increases cortisol secretion as a normal physiology. But in severe stress specially critical illness the cortisol production varies from high to low. Both high and low cortisol are associated with adverse outcome. Studies have shown that deficiency of cortisol secretion by the adrenal cortex increases morbidity and mortality in patients with septic shock. ${ }^{2}$ During stress of critical illness it is unclear how much cortisol level is necessary to survive. Moreover in

\section{Author \& Corresponding Author:}

Dr. S.M. Ashrafuzzaman, MD (Endocrinology), FACE (USA) Associate Professor, Department of Endocrinology, BIRDEM Room:1216, Birdem General Hospital (Level 12)

Email: ashraf_zaman1961@yahoo.com critically ill patients manifestations of adrenal insufficiency are numerous and nonspecific. So clinicians are urged to have a high index of suspicion and be alert to important diagnostic clues, such as hyponatremia, hyperkalemia, and hypotension, that are refractory to fluids and vasopressor without any clear causation ${ }^{3}$.

Few studies ${ }^{4}$ have focused on random cortisol level during critically illness. Level $>25 \mathrm{mcg} / \mathrm{dL}(695 \mathrm{nmol} / \mathrm{L})$ and level $<9 \mathrm{mcg} / \mathrm{dL}(250 \mathrm{nmol} / \mathrm{L})$ both are considered to have bad prognosis. Similarly it is also found that s. cortisol level vary among patients of septic shock. Moreover due to difference in cortisol binding globulin (CBG) total serum cortisol level may not reflect the ideal hypothalamic pituitary adrenal ( HPA) axis status. That's why some author suggest that free cortisol level more accurately reflects HPA axis activation ${ }^{5}$. In another study it is found that increase of serum cortisol level $<9 \mathrm{mcg} / \mathrm{dL}$ $(250 \mathrm{nmol} / \mathrm{L})$ from baseline, following intravenous corticotrophin (either at 30 minutes or 60 minutes) is associated with increased mortality from septic shock ${ }^{6,7}$.

The frequency and diagnosis of adrenal insufficiency in critically ill remain controversial despite studies demonstrating beneficial outcomes from treatment. Among the critically ill patients, septic shock has been associated with 
a greater than $50 \%$ mortality rate, ${ }^{8,9}$ and very little progress has been made 9,10 in improving mortality. Study showed beyond doubt that, administration of corticosteroids to adrenalectomized animals improved survival, ${ }^{11}$ similarly there is also proof that the continued suppression of adrenocortical function increased the mortality rate in critically ill patients ${ }^{12}$. So, there is chance of new onset adrenal insufficiency among critically ill subjects.

\section{Prevalence}

Adrenal insufficiency is estimated to occur at a rate of $0-30 \%$ in the critically ill population ${ }^{13,14}$ and may be as high as $25-40 \%$ in patients with septic shock, ${ }^{15,16}$ depending on the specific tests and threshold used to diagnose adrenal insufficiency, underlying disease, and severity of illness. Absolute adrenal insufficiency is rare among critically ill patients, with an incidence estimated to be $\leq 3$ percent ${ }^{25}$. However, observations argue that suboptimal cortisol production may be more common and associated with worse outcomes.

\section{Pathophysiology}

Three organs Hypothalamus, Anterior pituitary, and Adrenal cortex, collectively known as the hypothalamic - pituitary adrenal (HPA) axis maintain appropriate levels of glucocorticoid. There are three distinct modes of regulation of the HPA axis: diurnal rhythm in basal cortisol secretion, marked increases in steroidogenesis in response to stress, and the negative feedback regulation by adrenal cortisol. The mechanism of adrenal insufficiency during critical illness is not clear. However ACTH-cortisol dissociation has been reported during critical illness, referring to low circulating ACTH coinciding with elevated circulating cortisol ${ }^{3}$. Sometimes there is reduced cortisol metabolism leading to further hypercortisolemia. These changes usually are proportional to duration and degree of critical illness.

\section{Types of Adrenal Insufficiency}

Adrenal insufficiency can be categorized into two types: primary and secondary. Primary adrenal insufficiency, or Addison's disease, is caused by the inability of the adrenal gland to produce cortisol, aldosterone, or both, with the rest of the HPA axis remaining intact. Secondary adrenal insufficiency is caused by dysfunction of the hypothalamus, pituitary gland, or both (with a normal adrenal gland) and is characterized by low cortisol production and low or normal plasma ACTH concentration. The glucocorticoid deficiency and low ACTH concentration may result in hypotension and hyponatremia, with normal potassium and hydrogen ion concentrations. The ACTH-induced hyperpigmentation is absent in secondary adrenal insufficiency, and the release of aldosterone, sex hormones, and catecholamine is usually normal.

Adrenal insufficiency among critically ill patients is also known as, non functional or relative adrenal insufficiency, there is either insufficient cortisol or a cortisol level that may be high in absolute terms but insufficient to respond to the degree of stress. Thus, serum cortisol concentrations that are normal in well patients may be inappropriately low in severely sick patients. This inability to mount the appropriate response increases the risk of death during severe illness. ${ }^{17}$
Although absolute adrenal insufficiency is quite rare in critically ill patients, relative adrenal insufficiency is considerably more common. ${ }^{18}$ In chronic critical illness, adrenal insufficiency may also result from catecholamine receptor desensitization or down-regulation and/or chronic secretion of cytokines and other substances that suppress the HPA axis. This latter mechanism is sometimes called adrenal exhaustion syndrome. ${ }^{19}$

\section{Diagnosis of Adrenal Insufficiency in critically ill : High- versus Low-dose Adrenocorticotropic Hormone Stimulation Test}

For diagnosis of AI multiple tests have been developed, but the most commonly used test in the intensive care unit is the adrenocorticotropic hormone (ACTH) stimulation test. Rarely random or fasting s cortisol level is used. Still there is controversy about the level of S.Cortisol as cut off value for diagnosis of AI confidently or to select patient that who will be benefitted with steroid as management of AI among critically ill patients. Serum ACTH level usually not done to differentiate primary from secondary AI. Usually there are 2 types of ACTH stimulation tests, described below.

\section{Low dose (1 mcg) test}

Use of the low-dose test has been proposed by some clinicians. ${ }^{20,21}$ It is prepared by diluting the $250-\mu \mathrm{g}$ vial in $250 \mathrm{ml}$ of normal saline to obtain a concentration of corticotrophin (ACTH) $1 \mathrm{mcg} / \mathrm{ml}$ and using a $1-\mathrm{ml}$ syringe to accurately draw a dose. The syringes can be kept at $4^{\circ} \mathrm{C}$ for up to 4 months without any decline in biologic activity ${ }^{22}$. Results from a number of studies indicate that the low-dose test is more sensitive than the high-dose test for diagnosing adrenal insufficiency, but it is not as sensitive as the insulin tolerance test ${ }^{20,21,23,24}$. As low dose $(1 \mathrm{mcg})$ test is not well validated it is not yet recommended for critically ill patients by most of the authors. Till date serum cortisol with a minimum value $\geq 18 \mathrm{mcg} / \mathrm{dL}$ ( $500 \mathrm{nmol} / \mathrm{L})$ before or after ACTH injection $(1 \mathrm{mcg})$ is regarded as normal value (Excludes adrenal insufficiency, and proves adequate adrenal reserve, with or without stress or infection). The usual recommendation is that, Low dose test in noncritical illness subjects can be done on patients who recently developed ACTH or corticotrophin releasing hormone deficiency or who has recent pituitary surgery (Recent Secondary Adrenal Insufficiency). But its role in CIP is not evaluated.

\section{High dose $(250 \mathrm{mcg})$ test}

Measurement of cortisol levels with the High dose $(250 \mathrm{mcg})$ ACTH stimulation test is the standard and the most convenient test for diagnosing adrenal insufficiency in the ICU as well as non critical illness subjects. The test is based on the inability of a diseased adrenal gland to secrete adequate cortisol after injection of ACTH. The test is quick, simple, insensitive to interferences, reliable, and essentially free of adverse effects aside from very rare anaphylactic reaction. The test can be done anytime of the day even without fasting. Standard 3 samples 0,30 and 60 minutes are tested. Injection ACTH 250 mcg usually given IV. But in modified protocol IM can be given. Now a days mostly 3 sample is done, but very few author advised only one sample at 30 or 60 minutes. 
The current criteria used to indicate normal adrenal function are a minimum serum cortisol concentration $\geq 18$ to 20 $\mathrm{mcg} / \mathrm{dL}$ (500 to $550 \mathrm{nmol} / \mathrm{L}$ ) before or after corticotropin (ACTH) injection $(250 \mathrm{mcg})$.

A maximum increase of serum cortisol $\leq 9 \mathrm{mcg} / \mathrm{dL}$ from baseline, at 60 minutes after ACTH stimulation, is associated with increased mortality from septic shock..$^{24,25}$ Baseline and/or post-ACTH serum cortisol $<23.7 \mathrm{mcg} / \mathrm{dL}$ (670 nmol/L) predicts $\mathrm{AI}$ and responsiveness to exogenous steroids in septic shock (that is patient will Respond with steroid treatment). Responsiveness is defined by the ability to maintain a Mean Arterial Blood Pressure $>65 \mathrm{mmHg}$ without norepinephrine infusion within 24 hours after starting hydrocortisone ${ }^{26}$.

\section{Diagnosis}

Suboptimal cortisol production during septic shock has been termed "functional" adrenal insufficiency, "relative" adrenal insufficiency, or "critical illness-related corticosteroid insufficiency (CIRCI)". The functional adrenal insufficiency is usually transient. But level of basal or stimulated cortisol can't differentiate the absolute or functional adrenal insufficiency of critically ill patients, by their cut-off values. Broadly accepted consensus about diagnostic criteria for this entity is lacking. Response to ACTH testing should not be used to select patients for corticosteroid therapy. The ACTH stimulation test has failed to consistently identify patients with septic shock that benefit from corticosteroid use. This, together with the unreliability of available plasma cortisol assays in many labs suggest that ACTH stimulation testing is not clinically useful in distinguishing responders from not responders.

\section{Treatment}

Glucocorticoid has long been investigated as therapeutic agents in sepsis because the pathogenesis of sepsis involves an intense and potentially deleterious host inflammatory response. Two meta-analyses ${ }^{27,28}$ showed that use of glucocorticoid provides no overall benefit in the treatment of septic shock in all cases, with one of the meta-analyses suggesting that it may be harmful. ${ }^{28}$ The studies included in these meta-analyses used high-dose glucocorticoid for a short period of time. In fact, one meta-analysis ${ }^{27}$ showed no overall beneficial effect and no difference between low-dose and high-dose glucocorticoid.

Based on clinical evidence the surviving sepsis campaign (SSC) ${ }^{29,30}$ recommends to use IV hydrocortisone only in septic shock refractory to vasopressor and fluid resuscitation. The dose being 200-300 mg/day divided doses or by continuous drip for 5-7 days and withdraw gradually.

Evidence from randomized trials ${ }^{30,31}$ suggest that corticosteroid therapy is most likely to be beneficial in patients who have severe septic shock (defined as a systolic blood pressure $<90 \mathrm{mmHg}$ ) that is unresponsive to adequate fluid resuscitation and vasopressor administration. Data from ongoing clinical trials are needed to confirm that benefit.

In patients without shock, or patients with less severe septic shock (defined as those in whom fluid resuscitation and pressor therapy have restored hemodynamic stability), corticosteroid therapy does not appear to be beneficial. So corticosteroid is not recommended in these cases.

Close observation of those patients whose steroid therapy is stopped without being tapered is warranted. It is not recommended to add fludrocortisones to hydrocortisone.

\section{Summary and Recommendation}

Dysfunction of HPA axis with critical illness is best described by the term relative adrenal insufficiency (also known as critical illness related corticosteroid insufficiency) although the differentiation between absolute or relative adrenal insufficiency remains controversial. Prevalence varies according to disease, severity and duration.

In general at ICU, testing should only be employed in patients who have clinical features suggesting adrenal insufficiency (vasopressor dependent) or in those with severe sepsis or septic shock.

The current best diagnostic approach for adrenal insufficiency in all critically ill patients is random/basal cortisol measurement and peak response after $250 \mathrm{mcg}$ ACTH .

ACTH stimulation test should not be done in patients with no clinical features of adrenal insufficiency specially in patients with septic shock or ARDS to determine who will be treated with Steroid.

Serum Free Cortisol is not recommended in critically ill patients due to probable abnormality in Serum Albumin and Cortisol Binding Globulin (CBG).

A rational approach is to combine a lower cutoff limit of Serum cortisol $414 \mathrm{nmol} / \mathrm{L}(15 \mathrm{mcg} / \mathrm{dl})$ with a stimulated cortisol level 690nmol/L ( $25 \mathrm{mcg} / \mathrm{dl})$ which should reduce the possibility of missing either adrenal insufficiency among CIP. Some Intensive Care Unit uses cut-off value of S Cortisol from baseline after ACTH $250 \mathrm{mcg}$, that is, if increase of S Cortisol $<9 \mathrm{mcg} / \mathrm{dL}(250 \mathrm{nmol} / \mathrm{L})$ after Synacthen from baseline $\mathrm{S}$ cortisol (any level), it indicates Adrenal Insufficiency. Some Author believes that, this cutoff value may lead to some unnecessary treatment. So this cut-off value for diagnosis of AI among CIP to be weighed against the benefit along with clinical judgment.

ACTH stimulation test should not be used to identify adults with septic shock, who should receive hydrocortisone.

Moderate dose Hydrocortisone (100-200 mg/day) therapy is recommended in management strategy in patients with septic shock particularly if poor response with adequate fluid and vasopressor. Steroid should not be used for sepsis in absence of septic shock ${ }^{32}$. Dexamethason or other steroid for AI is not recommended.

Moderate dose of methylprednisolone can be considered in early severe ARDS and before day 14 in unresolved ARDS, but optimal duration of treatment is unclear.

If treated steroid should be tapered slowly, taper when vasopressor is no longer required.

The dose of Hydrocortisone should not exceed $50 \mathrm{mg} 6$ hourly (total $200 \mathrm{mg} /$ day in 4 divided doses). 
Alternate dosage regimen is $100 \mathrm{mg}$ IV bolus followed by 10 $\mathrm{mg}$ /hour IV infusion $(240 \mathrm{mg} / \mathrm{d})$. Some ICU recommends continuous flow of Hydrocortisone, rather than IV 6-8 hourly.

In early severe ARDS Methylprednisolone $1 \mathrm{mg} / \mathrm{kg} /$ day as continuous infusion.

Fludrocortisones $50-100 \mathrm{mcg}$ orally daily is usually necessary in Primary Adrenal Insufficiency.

Dexamethason is not recommended for Septic shock or ARDS.

\section{Conclusion}

Adrenal insufficiency can be life threatening particularly in stressful situations, and its diagnosis is always a challenge, especially in patients with critical illness. Since the condition appears to be common in patients with septic shock and rarely with ARDS, clinicians should have a high index of suspicion for its occurrence in critically ill patients with persistent hypotension despite adequate fluid resuscitation and/or poor hemodynamic response to vasopressor. Careful history taking and physical examination, complemented by other signs such as hyponatremia and hyperkalemia, should help identify most cases. Patients may have previous primary or secondary adrenal insufficiency but usually suffers from relative adrenal insufficiency of critical illness. Specialist in ICU and expert physicians working in ICU can suspect, diagnose and treat effectively for better outcome in critically ill with AI. Otherwise use of steroid in many patients in ICU may be harmful rather than beneficial.

\section{References}

1. Addison T. On the constitution and the local effects of diseases of the suprarenal capsules. London: Samuel Highly, 1855.

2. Zaloga GP, Marik P. Hypothalamic-pituitary-adrenal insufficiency. Crit Care Clin 2001;17:25-41.

3. Peeters B, Boonen E, Langouche L, Van den Berghe G. The HPA axis response to critical illness: New study results with diagnostic and therapeutic implications. Mol Cell Endocrinol. 2014 pii: S0303-7207(14)00368-2. doi:10.1016/j.mce.2014.11.012

4. Pandya U, Polite N, Lieber M. Increased total serum random cortisol levels predict mortality in critically ill trauma patients. Am Surg. 2014 Nov;80(11):1112-8.

5. Hamrahian AH, Oseni TS, Arafah BM. Measurements of serum free cortisol in critically ill patients. N Engl J Med 2004; 350:1629.

6. Annane D, Sébille V, Troché G, et al. A 3-level prognostic classification in septic shock based on cortisol levels and cortisol response to corticotropin. JAMA 2000; 283:1038.

7. Siraux V, De Backer D, Yalavatti G, et al. Relative adrenal insufficiency in patients with septic shock: comparison of low-dose and conventional corticotropin tests. Crit Care Med 2005; 33:2479.

8. Bone RC. The pathogenesis of shock. Ann Intern Med 1991;115:457-69.

9. Rockow EC, Astiz ME. The pathophysiology and treatment of septic shock. JAMA 1991;266:548-54.

10. Bone RC. A critical evaluation of new agents for the treatment of sepsis. JAMA 1991;266:1686-91.

11. Hinshaw LB, Bellar BK, Chang AC, et al. Corticosteroid/ antibiotic treatment of adrenalectomized dogs challenged with lethal E. coli. Circ Shock 1985:16:265-77.

12. Ledingham IMA, Watt I. Influence of sedation on mortality in critically ill multiple trauma patients [letter]. Lancet 1983;1:1270.
13. Knowlton AI. Adrenal insufficiency in the intensive care setting. J Intensive Care Med 1988;4:35-45.

14. Clark JL, Grossman AB. Adrenal insufficiency. In: DeGroot LJ, Jameson JL, eds. Endocrinology, vol 2, 5th ed. Philadelphia: Elsevier Saunders, 2005:2343-4.

15. Rothwell PM, Udwadia ZF, Lawler PG. Cortisol response to corticotropin and survival in septic shock. Lancet 1991;337:582-3.

16. Chin R. Adrenal crisis. Crit Care Clin 1991;7:23-42.

17. Ledingham IMA, Watt I. Influence of sedation on mortality in critically ill multiple traumapatients [letter]. Lancet 1983;1:1270.

18. Knowlton AI. Adrenal insufficiency in the intensive care setting. J Intensive Care Med 1988;4:35-45.

19. Clark JL, Grossman AB. Adrenal insufficiency. In: DeGroot LJ, Jameson JL, eds. Endocrinology, vol 2, 5th ed. Philadelphia: Elsevier Saunders, 2005:2343-4.

20. Abdu TAM, Elhadd TA, Neary R, Clayton RN. Comparison of the low dose short synachten test $(1 \mu \mathrm{g})$, the conventional dose $(250 \mu$ $\mathrm{g}$ ), and the insulin tolerance test for assessment of the hypothalamic-pituitary-adrenal axis in patients with pituitary disease. J Clin Endocrinol Metab 1999;84:838-43.

21. Rasmuson S, Olsson T, Hagg E. A low-dose ACTH test to assess the function of the hypothalamic-pituitary-adrenal axis. Clin Endocrinol (Oxf) 1996;44:151-6.

22. DicksteinG,AradE,ShechnerE. Low dose ACTH stimulation test.Endocrinologist1997; 7:285-93.

23. Mayenknecht J, Dieddderich S, Bahr V, Plockinger U, Oelkers M. Comparison of low-dose and high-dose corticotropin tests in patients with pituitary disease. J Clin Endocrinol Metab 1998;83:1558-62.

24. Siraux V, De Becker D, Yalavatti G, et al. Relative adrenal insufficiency in patients with septic shock: comparison of the low-dose and conventional corticotropin tests. Crit Care Med 2005;33:2479-86.

25. Rivers EP, Gaspari M, Saad GA, et al. Adrenal insufficiency in high-risk surgical ICU patients. Chest 2001;119:889-96.

26. Marik PE, Zaloga GP. Adrenal insufficiency during septic shock. Crit Care Med 2003; 31:141.

27. Lefering R, Neugebauer EA. Steroid controversy in sepsis and septic shock: a meta-analysis. Crit Care Med 1995;23: 1294-303.

28. Cronin L, Cook DJ, Carlet J, et al. Corticosteroid treatment for sepsis: a critical appraisal and meta-analysis of the literature. Crit Care Med 1995;23:1430-9.

29. Hossain Shahid Ferdous. Use of corticosteroid in critically ill: Cautions and precautions (Editorial).Bangladesh Crit Care J. September 2014; 2(2):51-52.

30. Annane D, Sebille V, Charpentier C,Bollaert PE, Francois B, Korach JM et al. Efeect of treatment of low doses hydrocortisone and fludrocortisone on mortality in patients with septic shock. JAMA 2002;288:862-71.

31. Sprung CL, Annane D, Keh D, Moreno R, Singer M, Freivogel K, Weiss YG, Benbenishty J, Kalenka A, Forst H, Laterre PF, Reinhart K, Cuthbertson BH, Payen D, Briegel J, CORTICUS Study Group. Hydrocortisone therapy for patients with septic shock. N Engl J Med. 2008;358(2):111.

32. Casserly B, Gerlach H, Phillips GS, Lemeshow S, Marshall JC, Osborn TM, Levy MM. Low-dose steroids in adult septic shock: results of the Surviving Sepsis Campaign. Intensive Care Med. 2012 Dec;38(12):1946-54. Epub 2012 Oct 12. 